\title{
Comorbidities and Quality of Life in Patients With Interferon-Refractory Chronic Hepatitis C
}

\author{
Robert J. Fontana, M.D., Cheryl A. Moyer, M.S., Seema Sonnad, Ph.D., Anna S. F. Lok, M.D., \\ Naomi Sneed-Pee, M.S., John Walsh, M.D., Steven Klein, M.D., and Steven Webster, M.D. \\ Department of Internal Medicine, Consortium for Health Outcomes Innovation and Cost-Effectiveness Studies \\ (CHOICES), Department of Surgery, University of Michigan Medical School, Huron Gastroenterology \\ Associates, Ann Arbor, Michigan; Botsford Hospital, Farmington Hills, Michigan; and Muskegon General \\ Hospital, Muskegon, Michigan
}

OBJECTIVES: Patients with chronic hepatitis C (HCV) consistently report a reduction in multiple domains of healthrelated quality of life (HRQOL) that does not correlate with liver disease severity. This may in part be due to the use of insensitive HRQOL instruments or extrahepatic factors that independently influence HRQOL. We hypothesized that a past history of substance abuse or active medical and psychiatric comorbidities would correlate with HRQOL scores.

METHODS: In 107 patients who had failed previous interferon therapy, HRQOL was measured by using the modified SF-36, a disease-specific instrument, and the Health Utilities Index (HUI) Mark III, a generic instrument.

RESULTS: Multiple SF-36 subscale and summary scores as well as the HUI Mark III attributes of emotion and pain were significantly reduced in the study population compared with healthy controls $(p<0.001)$. Serum alanine aminotransferase and HCV RNA levels, HCV genotype, liver histology, and $\mathrm{HCV}$ risk factors as well as demographic variables did not correlate with modified SF-36 and HUI scores. In addition, a history of alcohol abuse or dependency and intravenous drug use or dependency, identified in 52 and $51 \%$ of participants, respectively, did not correlate with HRQOL scores. However, the presence of one or more active medical comorbidities, defined as a chronic medical condition requiring treatment and monitoring, was significantly associated with both the modified SF-36 scores and HUI attribute deficits $(p<0.001)$. In particular, painful medical comorbidities or depressed mood requiring treatment were significantly associated with modified SF-36 scores and with HUI attribute deficits and utility scores $(p<0.001)$.

CONCLUSIONS: Active medical and psychiatric comorbidities may account for some of the reduction and variability in HRQOL scores in patients with chronic HCV who have failed previous interferon therapy. Future studies that control for the presence of active comorbidities in large groups of treatment naïve patients with varying severity of chronic $\mathrm{HCV}$ are needed to confirm these findings. (Am J Gastro- enterol 2001;96:170-178. (C) 2001 by Am. Coll. of Gastroenterology)

\section{INTRODUCTION}

An estimated 3-4 million Americans are chronically infected with hepatitis C (1-3). Clinical symptoms in chronic hepatitis $\mathrm{C}$ (CHC) tend to be mild, nonspecific, and intermittent, making it difficult to determine whether the reported symptoms are related to the liver disease itself or other extra-hepatic factors $(4,5)$. Nevertheless, patients with CHC report more frequent and severe physical symptoms, such as fatigue and malaise, compared with patients with other forms of chronic liver disease (5-7). Moderate impairments in multiple domains of health-related quality of life (HRQOL) have been reported in patients with CHC compared with healthy controls $(8-10)$. Several studies have failed to demonstrate a consistent relationship between HRQOL in patients with CHC and liver disease parameters such as serum ALAT (ALT) levels, quantitative hepatitis C virus (HCV) RNA levels, and liver histology (8-11). Nonetheless, HRQOL has been shown to significantly improve in patients who have a sustained virological response after treatment compared with virological relapsers and nonresponders (12-14). The lack of correlation between HRQOL and liver disease parameters in cross-sectional studies may be due to the use of insensitive generic HRQOL instruments that lack discriminative ability (11). In addition, failure to account for the impact of extrahepatic factors that may independently influence HRQOL may explain the lack of correlation.

The aim of this study was to determine whether variability in HRQOL scores in a cohort of patients with compensated $\mathrm{CHC}$ who had failed previous interferon (IFN) therapy could be explained in part by demographic and extrahepatic clinical variables. We hypothesized that patients with a history of substance abuse, including alcohol abuse or dependence and intravenous drug use (IDU) or dependence, would have reduced HRQOL scores compared with those without such a history because of impaired coping skills and 
frequent psychological disturbance (15-18). We also hypothesized that patients with active medical or psychiatric comorbidities such as diabetes and depression would have reduced HRQOL scores compared with subjects without these comorbidities in light of previous work illustrating the importance of comorbidities in HRQOL $(19,20)$. HRQOL was measured by using the modified SF-36 $(11,12,21)$, a disease-specific instrument developed for patients with CHC, and the Health Utilities Index (HUI) Mark II/III, a utility-based, generic HRQOL instrument (22). Administration of these two multidimensional and complementary HRQOL instruments allowed us to further characterize and compare the type and severity of HRQOL impairment in CHC patients.

\section{MATERIALS AND METHODS}

\section{Patient Population}

107 patients who had either relapsed or not responded to previous IFN therapy were studied. All patients had been off of IFN therapy for $\geq 3$ months and were being recruited for an investigational protocol of IFN- $\alpha 2 b$ and ribavirin (Rebetron, Schering-Plough Inc., Kenilworth, NJ). All subjects met the following inclusion criteria: age $>18 \mathrm{yr}$, serum ALT level above the upper limit of normal, detectable $\mathrm{HCV}$ RNA, previous treatment with IFN for $\geq 3$ months without a sustained virological response, and a liver biopsy obtained within the past 24 months. Exclusion criteria included active substance abuse within the past $2 \mathrm{yr}$ and severe or poorly controlled medical and psychiatric disorders that would preclude the use of IFN and ribavirin. A complete history and physical examination was performed by a single physician investigator (R.J.F.). The number and type of medical comorbidities, defined as medical conditions requiring active treatment and monitoring, was determined using methods similar to those of Kantz et al. (23). We also identified and recorded a subset of painful medical comorbidities known to be associated with prominent symptoms of bodily pain such as arthritis and low back pain. We obtained written informed consent from all study participants between September 1997 and April 1999 per local institutional review board guidelines.

\section{Confidential Survey}

All subjects completed a 56-item questionnaire detailing patient demographics, risk factors for acquiring $\mathrm{CHC}$, substance abuse history, and mental health history. Demographic data included patient age, sex, educational level, ethnic background, employment status, and marital status. CHC risk factors included blood transfusion before 1992, needle stick injuries, intravenous drug use, intranasal cocaine use, tattoo application, and high-risk sexual activity $(24,25)$. The estimated disease duration was determined by calculating the amount of time elapsed since the earliest identified parenteral risk factor exposure. In the 10 individuals without an identified $\mathrm{CHC}$ risk factor, we defined the duration of infection as the mean duration of infection in those with an identified $\mathrm{CHC}$ risk factor or factors.

Quantitative lifetime alcohol consumption was determined by using a modified version of the Skinner score (26). Periods of regular alcohol intake were determined by subject recall. The estimated amount of alcohol consumption during each period of consistent intake was calculated. Alcoholism or alcohol dependence were defined as existing in subjects who reported prior inpatient hospitalization, formal alcohol rehabilitation, and/or counseling for excessive alcohol consumption. Alcohol abuse was defined as individuals reporting affirmatively to one of the four CAGE questions when drinking in the past (27).

Quantitative lifetime exposure to intravenous drugs was determined by using a series of questions similar to that used for estimating alcohol consumption. The total number of days of intravenous drug use (IDU) was calculated from subject recall. Intravenous drug dependency was defined as individuals reporting prior drug rehabilitation or counseling, physical dependence, or daily drug use for $>30$ consecutive days (28).

Mental health history was determined from questions of previous emotional or mental health problems requiring treatment such as prescription medication or professional counseling. Individuals were classified as having a positive mental health history if they reported ever receiving treatment for a non-substance abuse-related mental health problem such as depressed mood or anxiety. The subjects receiving active treatment for a mood disorder at study enrollment were classified as having an active medical comorbidity.

\section{Liver Disease}

All subjects had compensated CHC with normal prothrombin time, serum albumin, and bilirubin levels. Serum ALT level was used as a surrogate marker of hepatic inflammation. Quantitative serum HCV RNA levels via reverse transcription-polymerase chain reaction and $\mathrm{HCV}$ genotypes were determined by a central reference laboratory using previously described methods (National Genetics Institute, Culver City, CA) (29, 30). Liver biopsies performed within 24 months of study entry were reviewed and classified as showing the presence or absence of cirrhosis.

\section{Health-Related Quality of Life}

A multidimensional and complementary assessment of HRQOL was obtained using the modified SF-36 and the HUI Mark II/III. Comparison of generic and disease-specific instrument results allows researchers to maximize the strengths of each instrument while minimizing each instrument's respective limitations (31).

\section{Modified SF-36.}

The modified SF-36, a 69-item questionnaire incorporating the original SF-36 and 6 additional hepatitis-specific subscales, was administered to all study subjects $(11,12,21$, 32). (The modified SF-36 is also known as the Hepatitis 
Quality of Life Questionnaire, QualityMetric, Inc., Lincoln, RI.) This instrument assesses generic functioning and wellbeing via multi-item scales measuring the following eight domains: physical functioning (PF), role physical (RP), bodily pain $(\mathrm{BP})$, general health $(\mathrm{GH})$, vitality $(\mathrm{V})$, social functioning (SF), role emotional (RE), and mental health $(\mathrm{MH})$. We also calculated physical and mental health summary scores (PCS, MCS). A CHC-specific module included questions addressing sleep, anxiety, depression, positive well-being, hepatitis limitations, and hepatitis distress. Original SF-36 subscale and summary scores were compared with those of healthy US population controls (33).

\section{Health Utilities Index}

The HUI Mark II/III was administered to all study participants. The HUI Mark II/III is a generic 17-item measurement of health status and HRQOL whose scoring is based upon population preferences for given health states (22). The Mark III system measures eight attributes of health status: vision, hearing, speech, emotion, cognition, pain, ambulation, and dexterity. Within each attribute of the HUI, there are multiple levels of functioning listed. A combination of levels of functioning across the attributes constitutes a given health state, which allows comparison with published Canadian population norms (34). The normative data for the instrument were obtained from a nationwide survey of 11, 924 Canadian households in 1991 in which 20,981 individuals over the age of 15 responded. The HUI Mark II/III also provides a utility score that specifies a value for any given health state. Although scores for both the HUI Mark II and Mark III were available for this study, the proportion of patients with attribute deficits was similar using the two instruments (data not shown). As a result, only the results for the HUI Mark III are presented.

\section{Statistical Methods}

Descriptive statistics were calculated and reported as the mean \pm SD unless indicated otherwise. Modified SF-36 and HUI Mark III scores were determined with Excel software, and statistical analysis was done with SPSS for Windows 1997 (SPSS Inc, Chicago, IL). Original SF-36 subscale and summary scores were compared with healthy US population controls using one-sample $t$ tests. Univariate analysis of associations among clinical variables of interest and the 10 subscales and summary scores of the original SF-36, the six subscales of the hepatitis-specific module of the SF-36, and the eight dimensions of the HUI Mark III were determined using correlations, $\chi^{2}$, and Kruskal-Wallis tests. Similarly, the individual results of the modified SF-36 and HUI Mark III were compared by using Kruskal-Wallis tests. Because of the limited sample size and the large number of clinical variables correlated with HRQOL scores, multiple regression analyses were not performed. A $p$ value of $<0.05$ was considered statistically significant.
Table 1. Clinical Characteristics of Study Population $(\mathrm{n}=107)$

\begin{tabular}{|c|c|}
\hline \multicolumn{2}{|l|}{ Demographics } \\
\hline Age $(y r)$ & $44.5 \pm 5.4$ \\
\hline$\%$ Caucasian & 94 \\
\hline$\%$ Male & 72 \\
\hline$\%$ Employed & 88 \\
\hline$\%$ Married & 75 \\
\hline$\%$ College graduate & 36 \\
\hline \multicolumn{2}{|l|}{ Liver disease } \\
\hline Estimated disease duration (yr) & $24 \pm 7$ \\
\hline Serum ALT (IU/L) & $154 \pm 126$ \\
\hline Ferritin $(\mathrm{ng} / \mathrm{ml})$ & $269 \pm 242$ \\
\hline $\operatorname{HCV}-R N A\left(\times 10^{6} \mathrm{eq} / \mathrm{ml}\right)$ & $3.63 \pm 1.79$ \\
\hline$\%$ Genotype 1 & 78 \\
\hline$\%$ Cirrhosis & 14 \\
\hline \multicolumn{2}{|l|}{$\mathrm{CHC}$ parenteral risk factors $(\mathrm{s})^{*}$} \\
\hline$\%$ Intranasal cocaine & 64 \\
\hline$\%$ Intravenous drugs & 51 \\
\hline$\%$ High-risk sexual behavior & 42 \\
\hline$\%$ Blood transfusion & 32 \\
\hline$\%$ Tattoo & 22 \\
\hline$\%$ Unknown & 9 \\
\hline \multicolumn{2}{|l|}{ Medical history } \\
\hline$\% \geq 1$ medical comorbidity & 57 \\
\hline$\% \geq 2$ medical comorbidities & 20 \\
\hline$\% \geq 1$ painful medical comorbidity & 21 \\
\hline$\%$ Positive mental health history $\dagger$ & 41 \\
\hline$\%$ Active depression $\ddagger$ & 21 \\
\hline$\%$ History of alcoholism or alcohol abuse & 52 \\
\hline \multicolumn{2}{|l|}{ Medical history } \\
\hline $\begin{array}{l}\text { Mean lifetime alcohol consumption in } \\
\text { individual drinks (range) }\end{array}$ & $\begin{array}{c}16,812 \pm 21,907 \\
(0-80,000)\end{array}$ \\
\hline$\%$ History of intravenous drug use & 51 \\
\hline$\%$ History of intravenous drug dependency & 18 \\
\hline $\begin{array}{l}\text { Mean lifetime intravenous drug use } \\
\text { in days (range) }\end{array}$ & $\begin{array}{r}407 \pm 1088 \\
(0-7200)\end{array}$ \\
\hline
\end{tabular}

\section{RESULTS}

\section{Study Population}

The majority of the 107 study participants were employed, married, Caucasian men (Table 1). The quantitative HCV RNA levels and distribution of HCV genotypes were comparable to that reported in other studies of US patients with CHC (2, 35). A minority of study subjects, $9 \%$, had no identifiable parenteral risk factor (24). The mean estimated duration of $\mathrm{CHC}$ infection was $24 \pm 7 \mathrm{yr}$.

Fifty-seven percent of the subjects had at least one active medical comorbidity, and $20 \%$ of subjects had more than two. Depressed mood (21\%), hypertension (14\%), and diabetes mellitus $(6 \%)$ were the most frequently identified active medical comorbidities (Table 2). Twenty percent of the study participants had an active medical comorbidity known to be associated with prominent symptoms of bodily pain (e.g., arthritis, migraine headache). A positive mental health history was identified in $42 \%$ of the study participants. Mental health disorders requiring current or past treatment included the following: depressed mood in 38 
Table 2. Active Medical Comorbidities in the Study Population $(\mathrm{n}=107)$

\begin{tabular}{lr}
\hline \multicolumn{1}{c}{ Condition } & no \\
\hline Depression & 22 \\
Hypertension & 15 \\
Arthritis* & 7 \\
Diabetes mellitus & 6 \\
Gastroesophageal reflux* & 5 \\
Back pain* & 4 \\
Migraine headaches* & 4 \\
Thyroid & 3 \\
Allergies & 2 \\
Anxiety & 2 \\
Asthma & 2 \\
Heart disease & 2 \\
Chronic bronchitis & 1 \\
Eczema & 1 \\
Essential tremor & 1 \\
Glaucoma & 1 \\
Morbid obesity & 1 \\
Pancreatic insufficiency & 1 \\
Neuropathy* & 1 \\
Irritable bowel* & 1
\end{tabular}

* Medical comorbidities were considered painful if the condition is commonly associated with significant bodily or somatic pain.

patients, depressed and anxious mood in 3 patients, anxious mood alone in 2 patients, and insomnia in 1 patient.

At the time of the study, $83 \%$ of the participants reported complete abstinence from alcohol, whereas $17 \%$ reported consuming a mean of $9.8 \pm 3.2$ drinks per week. However, $52 \%$ of study participants met criteria for a diagnosis of alcoholism or alcohol abuse in the past. Estimated lifetime alcohol consumption varied from 0 to 80,000 drinks and significantly correlated with a history of alcoholism or alcohol abuse $(p<0.001)$. Fifty-one percent of study participants related a history of previous IDU, but none of the study participants were actively using intravenous or illicit drugs at the time of the study. One-third of the 55 subjects with a past history of IDU met criteria for drug dependency. Quantitative lifetime IDU exposure positively correlated with a past history of IDU dependency $(p<0.001)$. Thirtysix percent of study participants reported a history of both alcohol abuse and IDU.

\section{Modified SF-36}

The study population had significantly lower scores than did healthy US population controls on all original SF-36 subscales except for the role emotional and the mental health summary scores (Fig. 1). CHC risk factors, quantitative alcohol consumption, alcoholism or alcohol abuse history, IDU history, and quantitative IDU exposure and dependency history were not significantly correlated with SF-36 scores. The patient demographic characteristics of age, education, and employment status also did not correlate with SF-36 scores. However, gender and marital status correlated with select SF-36 subscales: female gender with reduced physical functioning ( $p=0.049)$ and role emotional ( $p=$ 0.015); and single marital status with reduced mental health

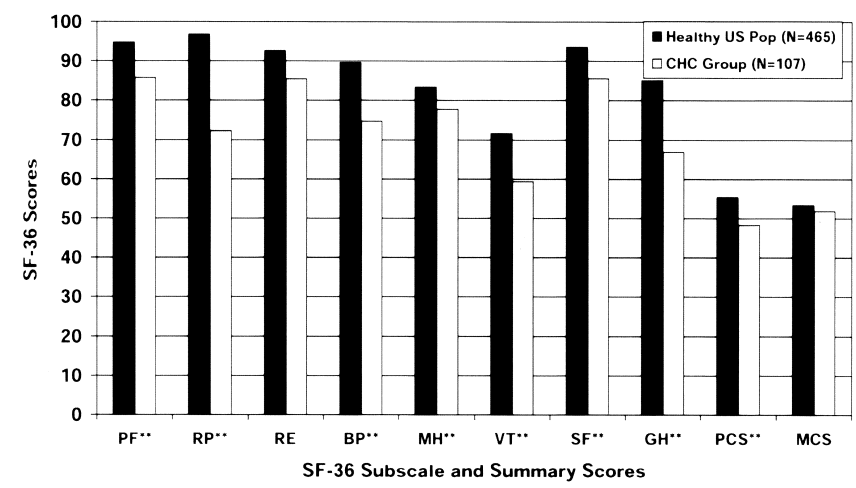

Figure 1. SF-36 subscale and summary scores in 107 patients with CHC compared with healthy US population controls (26). **p $<$ 0.001

( $p=0.027$ ). The hepatitis-specific subscales of the modified SF-36 did not correlate with alcoholism history, quantitative alcohol and IDU exposure, IDU dependency, CHC risk factors, or most demographic variables. However, the modified SF-36 dimension of positive well-being correlated positively with marital status $(p=0.041)$ and age $(p=0.043)$, and anxiety correlated with female gender $(p=0.049)$.

The liver disease parameters of serum ALT level, quantitative HCV RNA level, HCV genotype, and the presence or absence of cirrhosis demonstrated no correlation with original or hepatitis-specific SF-36 scores. By contrast, the presence and number of active medical comorbidities demonstrated strong correlations with SF-36 summary and subscale scores (Fig. 2). In particular, patients with painful medical comorbidities or depressed mood requiring treatment had lower modified SF-36 scores than did patients without either of these comorbidities (Table 3 ). The hepatitis-specific SF-36 subscale scores of sleep, hepatitis distress, and hepatitis limitations were also strongly correlated with the presence of medical comorbidities.

\section{HUI Mark III}

Thirteen percent of the study participants reported no deficits on the HUI Mark III, compared with $29 \%$ of healthy population controls $(p=0.001)$. Fifty-seven percent of the study population reported having two or more deficits on the HUI, compared with $33 \%$ of healthy population controls $(p=0.001)$. Deficits in vision, cognition, emotion, and pain were the most commonly affected attributes in the study population. The prevalence of emotional and pain deficits was significantly greater in the study population than in healthy population controls ( $p<0.001$ ). (Fig. 3) The frequent appearance of reduction in vision may reflect the fact that only adults were included in this study, whereas population data reflect those over age 15. In addition, visual acuity is known to decline with age (36). HUI deficits were not correlated with liver disease parameters, $\mathrm{CHC}$ risk factors, quantitative alcohol consumption, alcoholism history, IDU history and dependence, nor quantitative IDU exposure. The presence of medical comorbidities, however, was 

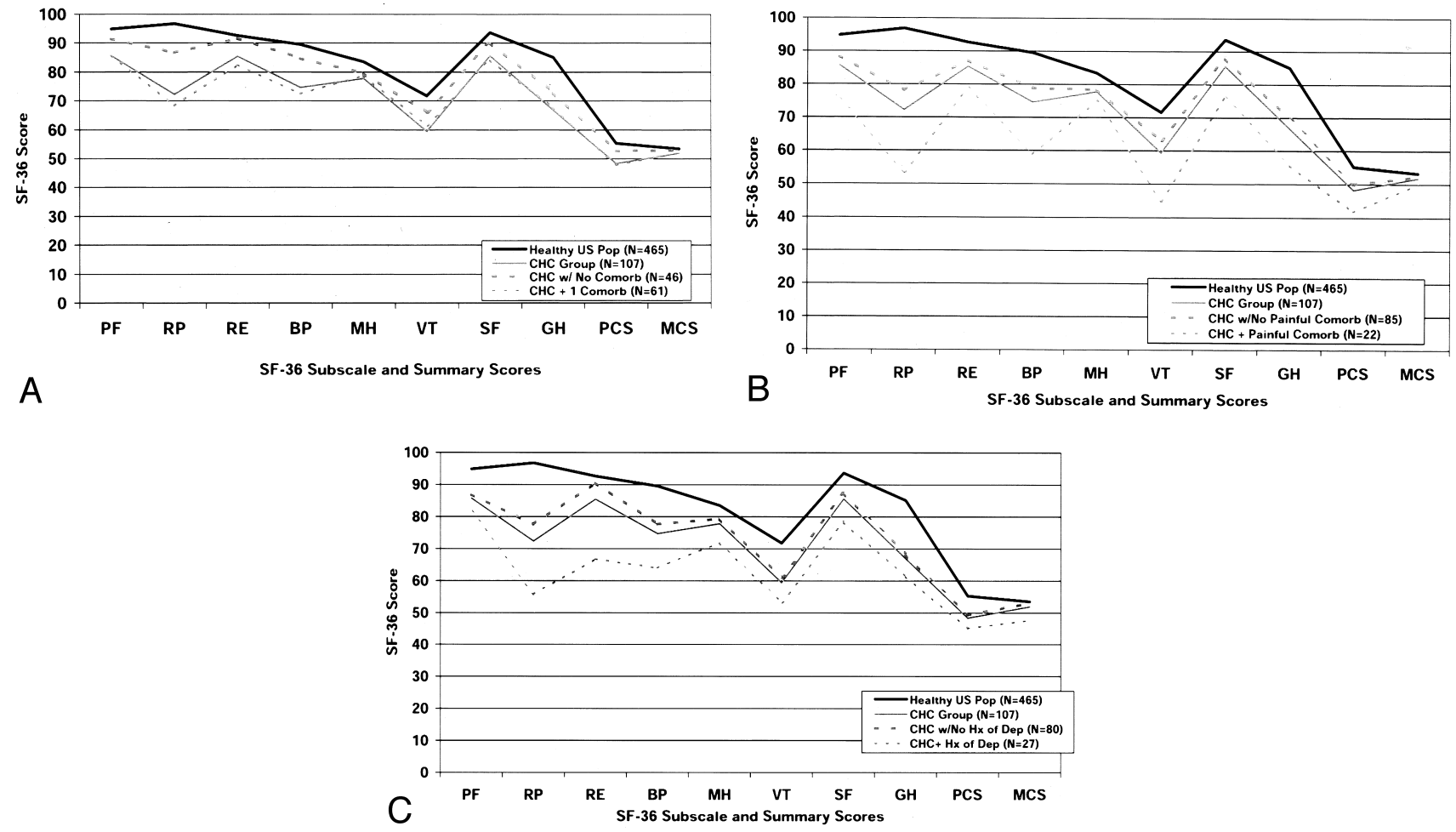

Figure 2. SF-36 subscale and summary scores in 107 patients with $\mathrm{CHC}$ compared with healthy population controls stratified by $(A)$ the presence and number of medical comorbidities, $(B)$ the presence of painful medical comorbidities, and $(C)$ the presence of depressed mood $(26,27)$.

significantly associated with HUI Mark III deficits in cognition $(p=0.038)$, dexterity $(p=0.002)$, speech $(p<$ $0.001)$, and pain $(p=0.006)$. The presence of painful medical comorbidities was associated with HUI deficits in ambulation $(p=0.012)$, vision $(p=0.02)$, and pain $(p<$ 0.001). As expected, a positive correlation between HUI cognitive deficits and depression was observed, but this trend did not reach statistical significance $(p=0.071)(37)$.
The mean HUI utility scores of the study population $(0.84 \pm$ $0.15)$ were significantly lower than those reported in the general population $(p<0.001)(38)$.

\section{Correlations Between the Modified SF-36 and HUI Mark III Scores}

Comparison of the HUI Mark III attribute scores and modified SF-36 subscale scores (Table 4) yielded many expected

Table 3. Correlation of Medical and Psychiatric Comorbidities With Modified SF-36 Subscale and Summary Scores

\section{A}

SF-36 Subscale and Summary Scales

Physical function

Role physical

Role emotional

Bodily pain

Mental health

Vitality

Social function

General health

Sleep

Hepatitis limitations

Hepatitis distress

Physical health summary score

Mental health summary score

\section{B}

Medical Comorbidities* $(\mathrm{N}=46)$

$<0.001$

0.001

0.046

0.004

0.56

0.003

0.016

0.012

0.05

0.003

0.121

$<0.001$

0.403

\section{$\mathrm{C}$}

Painful Medical

Comorbidities $\dagger$

$(\mathrm{N}=21)$

$\begin{array}{cc}<0.001 & 0.051 \\ 0.002 & 0.017 \\ 0.294 & 0.001 \\ 0.001 & 0.009 \\ 0.326 & 0.064 \\ <0.001 & 0.160 \\ 0.003 & 0.082 \\ 0.006 & 0.163 \\ 0.001 & 0.055 \\ <0.001 & 0.014 \\ 0.005 & 0.436 \\ <0.001 & 0.015 \\ 0.18 & 0.074\end{array}$

* $p$ values calculated by comparing column B and column A.

$\dagger p$ value calculated by comparing column $\mathrm{C}$ and column A.

$\ddagger p$ value colculated by comparing column $\mathrm{D}$ and column A. 


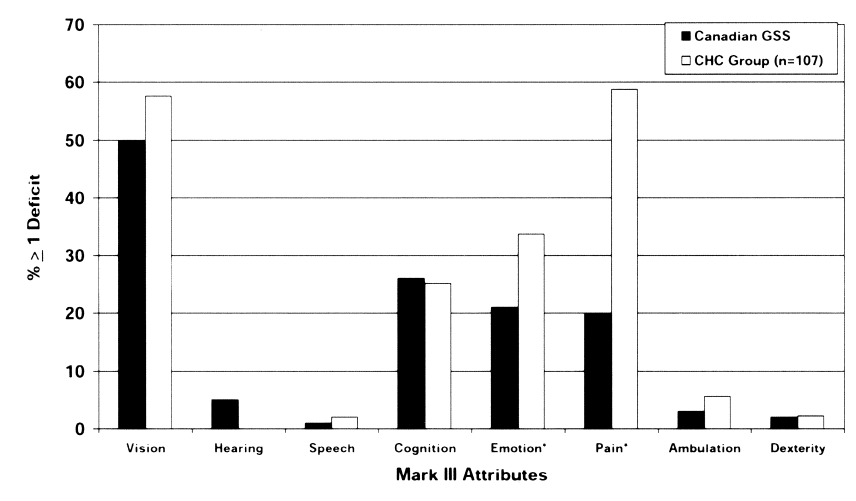

Figure 3. Comparison of the proportions of study patients and healthy population controls with one or more deficits on the HUI Mark III. The population control data were obtained from published tables of respondents to the General Social Survey in Canada in $1991(34) * p<.0 .05$.

associations, including the following: 1) HUI emotional deficits correlated with lower scores on the original SF-36 subscales of role physical, role emotional, mental health, vitality, social function, general health, and mental health summary scores as well as with the hepatitis-specific SF-36 subscales of anxiety, depression, positive well-being, and hepatitis distress; 2) HUI pain deficits correlated with lower scores on the modified SF-36 subscales of physical functioning, role physical, bodily pain, vitality, social functioning, general health, sleep, depression, hepatitis limitations, and physical health summary scores; and 3) HUI cognitive deficits correlated with lower scores on the modified SF-36 subscales of physical functioning, role physical, bodily pain, mental health, vitality, social functioning, general health, depression, positive well-being, hepatitis limitations, hepatitis distress, and the mental health summary score. (Table 3) In addition, HUI utility scores significantly correlated with all of the modified SF-36 subscales $(p<0.01)$. However, some of the correlations between the HUI Mark III attributes and modified SF-36 scores were unexpected. For example, the SF-36 physical health summary scores correlated with the HUI deficits of cognition and emotion. In addition, the hepatitis distress and hepatitis limitation scores were strongly associated with the HUI dimensions of ambulation, cognition, emotion, and pain.

\section{DISCUSSION}

CHC is frequently diagnosed in asymptomatic individuals coming under medical attention for incidental reasons or in patients presenting with nonspecific symptoms of fatigue and abdominal pain. Although it is difficult to establish a relationship between the presence and severity of compensated $\mathrm{CHC}$ and clinical symptoms, patients with $\mathrm{CHC}$ consistently report moderate impairments in multiple domains of HRQOL $(8-11)$. The aim of this study was to identify clinical factors that may in part explain some of the reported impairments and variability in HRQOL in ambulatory patients with compensated CHC. In this study of $107 \mathrm{CHC}$ patients who failed previous IFN therapy, original SF-36 subscale and summary scores were significantly reduced compared with those of healthy population controls (Fig. 1). The magnitude of reduction in HRQOL scores was comparable to that seen in patients with other uncomplicated chronic medical illness such as diabetes mellitus and hypertension (39).

Our study population may not be representative of the full spectrum of patients with CHC. The patients were being screened for participation in a clinical trial at a tertiary care center. As such, they may have had more symptomatic or severe liver disease. In addition, all of these patients had failed previous IFN therapy, which may have influenced their health status perception and sense of well-being. As a result, these patients may represent a subgroup of $\mathrm{CHC}$ patients with more advanced liver disease or other complicating issues. Together, these factors would suggest that the HRQOL scores of our study population may overestimate the impact of CHC on HRQOL. However, when comparing the level of impairment in the modified SF-36 scores to other published studies involving larger numbers of patients as well as treatment-naive patients, the quality and quantity of HRQOL impairment were similar $(10,11)$. In addition, the high prevalence of substance abuse, identified $\mathrm{CHC}$ risk factors, and disease duration in our study population is similar to what has been reported in other studies (10). Therefore, our study population is presumably comparable to that reported in other studies of HRQOL in patients with CHC.

As in other reports, we failed to demonstrate a relationship between the original and hepatitis-specific SF-36 subscale and summary scores and several liver disease parameters (i.e., serum ALT, HCV RNA, HCV genotype, presence of cirrhosis) (8-11). Similarly, there seemed to be no relationship between HUI deficits and liver disease parameters. It should be kept in mind, however, that serum ALT is not a reliable predictor of the severity of histological hepatitis in CHC $(40,41)$. Nonetheless, these observations

Table 4. Correlation of HUI Mark III Attributes and Utility Score With Modified SF-36 Subscale and Summary Scores

\begin{tabular}{lrrrrrrrrrrrrr}
\hline \multicolumn{1}{c}{ HUI } & \multicolumn{1}{c}{ PF } & \multicolumn{1}{c}{ RP } & \multicolumn{1}{c}{ RE } & \multicolumn{1}{c}{ BP } & \multicolumn{1}{c}{ MH } & \multicolumn{1}{c}{ VT } & \multicolumn{1}{c}{ SF } & \multicolumn{1}{c}{ GH } & PWB & HepLim & HepDis & PCS & MCS \\
\hline Cognition & 0.002 & $<0.001$ & 0.021 & 0.003 & 0.001 & $<0.001$ & $<0.001$ & 0.001 & $<0.001$ & $<0.001$ & $<0.001$ & $<0.001$ & 0.007 \\
Emotion & 0.678 & 0.001 & $<0.001$ & 0.381 & $<0.001$ & $<0.001$ & $<0.001$ & $<0.001$ & $<0.001$ & 0.012 & 0.001 & 0.417 & $<0.001$ \\
Pain & $<0.001$ & $<0.001$ & 0.119 & $<0.001$ & 0.021 & 0.001 & $<0.001$ & $<0.001$ & 0.02 & $<0.001$ & 0.004 & $<0.001$ & 0.141 \\
Utility & 0.002 & $<0.001$ & $<0.001$ & $<0.001$ & $<0.001$ & $<0.001$ & $<0.001$ & $<0.001$ & $<0.001$ & $<0.001$ & $<0.001$ & $<0.001$ & $<0.001$ \\
\hline
\end{tabular}

$\mathrm{PF}=$ physical function; $\mathrm{RP}=$ role physical; $\mathrm{RE}=$ role emotional; $\mathrm{BP}=$ bodily pain; $\mathrm{MH}=$ mental health; $\mathrm{VT}=$ vitality; $\mathrm{SF}=$ social function; $\mathrm{GH}=$ general health; $\mathrm{PWB}$ = positive well-being; HepLim = hepatitis limitations; HepDis = hepatitis distress; PCS = physical health summary score; MCS = mental health summary score. 
suggest that extrahepatic factors may play an important role in determining HRQOL in CHC.

Demographic variables of age, educational level, and employment status did not explain the variability in the modified SF-36 subscale and summary scores. This may in part be due to the limited heterogeneity in these variables in our study population (Table 1). Contrary to our hypothesis, a past history of alcoholism or alcohol abuse identified in $52 \%$ of study subjects did not correlate with modified SF-36 scores or with HUI deficits. This finding is somewhat surprising in light of previous work demonstrating that alcoholics have impaired coping skills and a higher incidence of psychopathology, which we hypothesized would lead to lower HRQOL scores $(15,16)$. In addition, a history of IDU or intravenous drug dependency did not correlate with modified SF-36 scores or HUI deficits. This finding was also unexpected because previous studies have shown that subjects with a history of IDU have psychological disturbances that may lead to a reduction in HRQOL $(17,18,42,43)$. Furthermore, a recent study suggested that $\mathrm{CHC}$ patients with a history of IDU have a trend toward reduced HRQOL compared with subjects who acquired $\mathrm{CHC}$ through other means (10). It is possible that the extended period of time that elapsed from pathological alcohol consumption and IDU exposure to the current study (alcohol $=6.0 \pm 4.9 \mathrm{yrs}$; IDU $=17.6 \pm 7.6 \mathrm{yrs}$ ) may explain the lack of correlation of these clinical variables with HRQOL scores.

The clinical variables that correlated the best with modified SF-36 scores were the presence of psychiatric and medical comorbidities, particularly painful medical comorbidities (Table 3). Medical comorbidities also strongly correlated with HUI scores. The prevalence and type of medical comorbidities identified in our study population are comparable to those reported in other large surveys of ambulatory medical patients (19). Previous studies have demonstrated that the type and number of medical comorbidities are one of the strongest correlates of HRQOL scores $(19,20)$. Furthermore, studies have demonstrated that painful medical comorbidities such as low back pain or arthritis have the greatest impact on HRQOL (44). Recently, the cost of care and resource utilization in patients with compensated CHC has been linked to the presence of medical comorbidities (45).

A non-substance abuse-related mental health history and, in particular, depressed mood requiring active treatment, also strongly correlated with HRQOL scores as expected (Table 3, Fig. 2). Thirty-eight percent of the study participants had a positive mental health history due to depression, and $21 \%$ were receiving treatment for depressed mood. The prevalence of depressed mood in our population is similar to that reported in studies of outpatients with other chronic illnesses and in patients with CHC (46-50). The high prevalence of depression in patients with $\mathrm{CHC}$ may in part be due to the route of infection, the psychological burden of a chronic illness, or the liver disease itself (46, 51). One recent study reported that individuals who knew they were seropositive for CHC had lower HRQOL scores than did subjects who were unaware of their serostatus (52). These findings suggest that the psychological impact or worry of being diagnosed with $\mathrm{CHC}$ is substantial. Our data demonstrate that depressed mood is an important clinical correlate of HRQOL in patients with CHC $(45,53)$.

HUI Mark III deficits in bodily pain and emotional attributes were more common in patients with $\mathrm{CHC}$ compared with healthy population controls (Fig. 3). HUI utility scores were also significantly lower in the study group compared with population controls $(p<0.001)$. The level of reduction in HUI utility scores corresponds to the magnitude in reduction reported in patients with asthma or early HIV infection $(54,55)$. Utility scores were not significantly associated with patient demographics, liver disease severity, substance abuse history, or mental health history. However, medical comorbidities $(p<0.001)$, especially painful medical comorbidities $(p<0.001)$, were significantly associated with lower utility scores among study patients (Data not shown).

It is possible that the HRQOL instruments selected for this study may not be adequately sensitive to detect meaningful differences in HRQOL scores in patients with varying severity of CHC. For example, the HUI Mark III is a generic instrument and has not been previously validated in patients with $\mathrm{CHC}$, although it has been studied and validated in other conditions $(54,56)$. Similarly, the SF-36 is a generic instrument that may lack disease specificity in CHC (11). However, the modified SF-36 provides supplemental information in several generic domains as well as in the diseasespecific domains of hepatitis distress and hepatitis limitations and has been shown to be psychometrically valid $(11,21)$. Two large longitudinal studies using the modified SF-36 have shown that HRQOL scores improve to the level of those of healthy controls after viral eradication, suggesting that it is a valid and sensitive instrument for measuring HRQOL in patients with CHC $(13,14)$. In addition, our study demonstrated consistent reductions in HRQOL scores across the SF-36 subscale and summary scores, the disease-specific SF-36 subscale scores, and the HUI scores, suggesting that the instruments used and the relationships observed are valid in this patient population. Furthermore, comparison of HUI Mark III scores with SF-36 scores demonstrated many expected associations (Table 4).

In summary, substance abuse history did not correlate with HRQOL scores. However, as hypothesized, the presence and number of active medical and psychiatric comorbidities were a strong predictor of HRQOL scores. We conclude that future studies that control for the presence of medical and psychiatric comorbidities in large groups of CHC patients with varying severity of liver disease are needed to confirm our findings and improve our understanding of the determinants of HRQOL in CHC. 


\section{ACKNOWLEDGMENTS}

This work was supported in part by an unrestricted educational grant from Schering-Plough, Inc., Kenilworth, NJ. We thank the study coordinators at each participating site for their assistance with data collection and management: Michelle Chandler, R.N., Theresa Niger, R.N., and Suzanne Sutton, R.N.

\section{Reprint requests and correspondence: Robert J. Fontana, M.D., University of Michigan Medical Center, 3912 Taubman Center, Box 0362, Ann Arbor, MI 48109.}

Received Mar. 22, 2000; accepted Aug. 25, 2000.

\section{REFERENCES}

1. Seeff LB. Natural history of hepatitis C. Hepatology 1997; 26(suppl):21S-28S.

2. Alter MJ, Kruszon-Moran D, Nainan OV, et al. The prevalence of hepatitis C virus infection in the United States, 1988 through 1994. N Engl J Med 1999;341:556-562.

3. National Institutes of Health Consensus Development Conference Panel Statement: Management of hepatitis C. Hepatology 1997;26(suppl):2S-10S.

4. Villano SA, Vlahove D, Nelson KE, et al. Persistence of viremia and the importance of long term follow-up after acute hepatitis C infection. Hepatology 1999;29:908-14.

5. Hoofnagle JH. Hepatitis C. The clinical spectrum of disease. Hepatology 1997;26(suppl):15S-20S.

6. Barkhuizen A, Rosen HR, Wolf S, et al. Musculoskeletal pain and fatigue are associated with chronic hepatitis C. Am J Gastroenterol 1999;94:1355-60.

7. Koff RS, Dienstag JL. Extrahepatic manifestations of hepatitis $\mathrm{C}$ and the association with alcoholic liver disease. Semin Liver Dis 1995;15:101-9.

8. Davis GL, Balart LA, Schiff ER, et al. Assessing HRQOL in chronic hepatitis C using the SIP. Clin Ther 1994;16:334-43.

9. Carithers RL, Sugano D, Bayliss M. Health assessment for chronic HCV. Dig Dis Sci 1996;41(suppl):75S-80S.

10. Foster GR, Goldin RD, Thomas HC. Chronic Hepatitis C virus infection causes a significant reduction in HRQOL in the absence of cirrhosis. Hepatology 1998;27:209-12.

11. Bayliss MS, Gandek B, Bungay KM, et al. A questionnaire to assess the generic and disease-specific health outcomes of patients with chronic Hepatitis C. Qual Life Res 1998;7:3955.

12. Bonkovsky HL, Wooley JM, the Consensus IFN Study Group. Reduction of HRQOL in chronic hepatitis $\mathrm{C}$ and improvement with IFN therapy. Hepatology 1999;29:264-70.

13. Ware JE, Bayliss MS, Mannocchia M, the International Hepatitis Interventional Therapy Group. Health-related quality of life in chronic hepatitis C: Impact of disease and treatment response. Hepatology 1999;30:550-5.

14. Neary MP, Cort S, Bayliss MS, et al. Sustained virologic response is associated with improved health-related quality of life in relapsed chronic hepatitis C patients. Semin Liv Dis 1999;19(suppl 1):77S-85S.

15. Cannon DS, Bell WE, Fowler DR, et al. MMPI differences between alcoholics, and drug abusers. Psychol Assess 1990; 3:51-5.

16. Hesselbroack MN, Meyer RE, Keener JJ. Psychopathology in hospitalized alcoholics. Arch Gen Psychiatry 1985;42: 1050-5.

17. Kendall J, Sherman M, Bigelow G. Psychiatric symptoms in polysubstance abusers: Relationship to race, age, sex and age. Addict Behav 1995;20:685-90.

18. Lipsitz J, Williams J, Rabkin J, et al. Psychopathology in male and female intravenous drug users with and without HIV infection. Am J Psychiatry 1994;151:1662-8.

19. Stewart AL, Greenfield S, Hays RD, et al. Functional status and well-being of patients with chronic conditions. JAMA 1989;262:907-13.

20. Ware JE, Sherbourne CD. The MOS 36-item short-form health survey (SF-36). Med Care 1992;30:473-83.

21. Bayliss MS. Methods in outcomes research in hepatology: Definitions and domains of quality of life. Hepatology 1999; 29(suppl 1):7S-8S.

22. Feeny D, Furlong W, Boyle M, et al. Multi-attribute health status classification systems. Pharmacoeconomics 1995;7: 490-502.

23. Kantz ME, Harris WJ, Levitsky K, et al. Methods for assessing condition-specific and generic functional status outcomes after total knee replacement. Med Care 1992;30:240-52.

24. Alter MJ. Epidemiology of Hepatitis C. Hepatology 1997; 26(suppl):62S-5S.

25. Conry-Cantilena C, VanRaden M, Gibble J, et al. Routes of infection, viremia, and liver disease in blood donors found to have hepatitis C infection. N Engl J Med 1996;334:1691-6.

26. Skinner HA, Sheu WJ. Reliability of alcohol use indices: Lifetime drinking history and MAST. J Stud Alcohol 1982; 43:1157-70.

27. Ewing JA. Detecting alcoholism, the CAGE questionnaire. JAMA 1984;252:1905-7.

28. Gavin D, Ross H, Skinner H. Diagnostic accuracy of the drug abuse screening test. Br J Addict 1989;84:301-5.

29. Pockros PJ, Bain VG, Hunter EB, et al.. A comparison of reverse transcription-polymerase chain reaction and branchedchain DNA assays for hepatitis C virus RNA in patients receiving Interferon treatment. J Viral Hepatitis 1999;6:14550 .

30. Stuvyer L, Rossau R, Wyseur A, et al. Typing of hepatitis C virus isolates and characterization of new genotypes. J Gen Virol 1993;74:1093-102.

31. Moyer CA, Fendrick AM. Measuring health-related quality of life in patients with upper gastrointestinal disease. Dig Dis 1998;16:315-24.

32. Ware JE, Sherbourne C. The MOS 36-item short-form health survey. Conceptual framework and item selection. Med Care 1992;30:473-83.

33. Ware JE, Kosinski M, Bayliss MS, et al. Comparison of methods for the scoring and statitistical analysis of the SF-36 health profiles and summary measures: Summary of results from the Medical Outcomes Study. Med Care 1995;33(suppl 4):AS264-AS279.

34. Statistics Canada General Social Survey Analysis Series. Health Status of Canada. Catalogue 11-612E No 8, Ottawa, Canada, 1991.

35. Mahaney K, Tedeschi V, Maertens G, et al. Genotypic analysis of hepatitis $C$ virus in American patients. Hepatology 1994;20:1405-1411.

36. Schachat AP. Common problems associated with impaired vision. In: Barker LR, Burton JR, Zieve PD, eds. Principles of ambulatory medicine, 4th ed. Philadelphia: Williams \& Wilkins, 1995:1415-22.

37. Grant I, Adams KM. Neuropsychological assessment of neuropsychiatric disorders. New York: Oxford Press, 1996:20017.

38. Boyle MH, Fulong W, Feeny D, et al. Reliability of the Health Utilities Index-Mark III used in the 1991 cycle 6 Canadian General Social Survey Health Questionnaire. Qual Life Res 1995;4:249-57. 
39. McHorney CA, Ware JE, Faczek AE. The MOS 36-Item Short from Health Survey (SF-36): Psychometric and clinical tests of validity in measuring physical and mental health constructs. Med Care 1993;31:247-63.

40. Haber MM, West AB, Haber AD, et al. Relationship of aminotransferases to liver histological status in chronic hepatitis C. Am J Gastroenterol 1995;90:1250-7.

41. McCormick SE, Goodman ZD, Maydonovitch CL, et al. Evaluation of liver histology, ALT elevation, and HCV RNA titer in patients with chronic hepatitis C. Am J Gastroenterol 1996; 91:1516-22.

42. Rounsaville BJ, Weissman MM, Kleber H, et al. Heterogeneity of psychiatric diagnosis in treated opiate addicts. Arch Gen Psychiatry 1982;39:161-6.

43. Dinwiddie SH, Reich T, Clininger RC. Psychiatric comorbidity and suicidality among intravenous drug users. J Clin Psychiatry 1992;53:364-9.

44. Xuan J, Kirchdoerfer LJ, Boyer JG, et al. Effects of comorbidity on health-related quality of life scores: An analysis of clinical trial data. Clin Ther 1999;2192:383-403.

45. Pauly MP, Ready J, Szpakowski J, et al. Increased cost of care in hepatitis $\mathrm{C}$ is due to comorbid medical conditions until the development of decompensated cirrhosis. Hepatology 1999; 30:A167 (abstract).

46. Nielsen AR, Williams TA. Depression in ambulatory medical patients. Arch Gen Psychiatry 1980;37:999-1004.

47. Mulrow CD, Williams JW, Gerety MB, et al. Case-finding instruments for depression in primary care settings. Ann Intern Med 1995;122:913-21.

48. Raft D, Davidson J, Toomey TC, et al. Inpatient and outpatient patterns of psychotropic drug prescribing by nonpsychiatric physicians. Am J Psychiatry 1975;132:1309-12.

49. Hunt CM, Dominitz JA, Bute BP, et al. Effect of interferon-a treatment of chronic hepatitis $\mathrm{C}$ on health related quality of life. Dig Dis Sci 1997;42:2482-6.

50. Johnson ME, Fisher DG, Fenaughty A, et al. Hepatitis C virus, and depression in drug users. Am J Gastroenterol 1998;93: 785-89.

51. Koff RS. Impaired health-related quality of life in chronic hepaitits C: The how but not the why. Hepatology 1999;29: 177-279.

52. Rodger AJ, Jolley D, Thompson SC, et al. The impact of diagnosis of hepatitis $\mathrm{C}$ virus on quality of life. Hepatology 1999;30:1299-301.

53. Karlsen KH, Larsen JP, Tandberg E, et al. Influence of clinical and demographic variables on quality of life in patients with Parkinson's disease. J Neurol Neurosurg Psychiatry 1999;66: 431-5.

54. Revicki DA, Leidy NK, Brenna-Diemer F, et al. Integrating patient preferences into health outcomes assessment. The multi-attribute asthma symptom utility index. Chest 1998;114: 998-1007.

55. Owens DK, Cardinalli AB, Nease RF. Physician's assessments of the utility of health states associated with human immunodeficiency virus (HIV) and hepatitis B virus (HBV) infection. Qual Life Res 1997;6:77-86.

56. Mathias SD, Bates MM, Pasta DJ, et al. Use of the health utilities index with stroke patients and their caregivers. Stroke 1997;28:1888-94. 